\title{
VANTAGGI DELLA CONFORMITÀ E DELLA GESTIONE DEL RISCHIO
}

\section{ARTICOLO ORIGINALE}

SILVA, Lilian Reis da ${ }^{1}$

SILVA, Lilian Reis da. Vantaggi della conformità e della gestione del rischio. Revista Científica Multidisciplinar Núcleo do Conhecimento. Anno. 06, Ed. 12, Vol. 04, pp. 123-147. Dicembre 2021. ISSN: 2448-0959, Link di accesso: https://www.nucleodoconhecimento.com.br/economia-aziendale/vantaggi-dellaconformita, $\quad$ DOI: $\quad 10.32749$ /nucleodoconhecimento.com.br/economiaaziendale/vantaggi-della-conformita

\section{RIEPILOGO}

La conformità è un programma che mira a proteggere le organizzazioni dal verificarsi di frodi finanziarie, corruzione, comportamenti e/o comportamenti inappropriati dei dipendenti ad esse collegati, prevenendo che la loro buona reputazione e solidità finanziaria vengano compromesse. È uno strumento il cui scopo è stabilire regole, standard e linee guida per i processi interni all'interno delle organizzazioni. È stato sviluppato negli Stati Uniti nel 1970 e le sue pratiche sono state trasformate in un istituto legale, attraverso l'emanazione della legge pionieristica FCPA (Foreign Corrupt Practices Act), motivata dal caso Watergate, che ha coinvolto l'allora presidente Richard Nixon e membri del suo governo, pagando tangenti in ricerca a favore della sua rielezione. In questo contesto, questo articolo ha come domanda guida: come collaborano i vantaggi offerti dal programma di conformità e Gestione del rischio per ridurre i rischi di frode, atti illeciti e corruzione all'interno delle organizzazioni? L'obiettivo di questo studio era presentare i benefici forniti dall'adozione del programma di conformità e Gestione del rischio nelle aziende pubbliche e private, nei loro processi interni e nelle relazioni con il loro segmento di attività, e come collaborano per mitigare le frodi. La ricerca bibliografica è stata adottata come metodologia,

\footnotetext{
${ }^{1}$ Post-laurea in gestione aziendale, economista e tecnica contabile.
}

RC: 103918

Disponibile in: https://www.nucleodoconhecimento.com.br/economiaaziendale/vantaggi-della-conformita 
discutendo i benefici causati dai programmi di prevenzione delle frodi. È stato riscontrato che i programmi di conformità e di gestione del rischio apportano benefici effettivi di protezione del rischio, mitigando le frodi e la corruzione, insieme a nuove soluzioni di governance delle tecnologie dell'informazione (IT), come la Enterprise Governance, Risk Management and Compliance (EGRC).

Parole chiave: Frode all'interno delle organizzazioni, Prevenzione dei rischi di corruzione, Conformità, Compliance.

\section{INTRODUZIONE}

Discutere i vantaggi della conformità e della gestione dei rischi implica la presentazione di concetti su cosa sia un programma di conformità e quali siano i rischi che le aziende corrono quando non riescono ad adottare questo tipo di programma, in considerazione dei diversi tipi di frode, tra cui corruzione, riciclaggio di denaro, manipolazioni con informazioni interne e / o quando i dipendenti commettono azioni che possono danneggiare l'immagine di un'organizzazione, può anche causare perdite finanziarie.

Secondo Michelotti (2020, p. 6), l'adozione di un programma di conformità e risk management fornisce trasparenza alle organizzazioni, dimostrando l'esistenza di una condotta etica; inoltre, collabora con le pratiche di buona Corporate Governance, che è "l'insieme dei processi attraverso i quali le aziende sono gestite".

Secondo Sobreira Filho, Leite e Martins (2019, p. 23), "il programma di conformità è uno strumento aziendale la cui metodologia per la sua implementazione si basa sull'etica aziendale e sull'integrità come il suo più grande valore".

II termine conformità (chiamato anche compliance), nato dal verbo inglese "to comply", riassunto da Coelho e Santos Jr (2021, p. 8) come "soddisfare le imposizioni dell'ordinamento giuridico o dell'ordine interno dell'azienda" (grifoni dell'originale). A questo concetto, Gonsales (2016) aggiunge che "se all'inizio, conformità significava 
"seguire le regole", dalle grandi indagini brasiliane, è venuto anche a significare "seguire la legge anticorruzione".

È interessante notare che la conformità si applica alle aziende pubbliche o private, di diverse dimensioni e segmenti, situate in tutti i paesi, organizzazioni che hanno il desiderio di agire con etica, morale e nella promozione dello sviluppo umano, sociale, economico e finanziario.

Questo articolo è stato sviluppato come ricerca bibliografica, per la quale è stata definita la seguente domanda: in che modo i benefici offerti dal programma conformità e Gestione del rischio collaborano per ridurre i rischi di frode, illecito e corruzione all'interno delle organizzazioni? A sua volta, l'obiettivo generale è stato quello di presentare i benefici forniti dall'adozione del programma di conformità e Gestione del rischio nelle aziende pubbliche e private, nei loro processi interni e nelle relazioni con il loro segmento di attività, e come collaborano per la mitigazione delle frodi.

\section{CONFORMITÀ}

\subsection{CONCETTO}

Affinché un'azienda sia considerata affidabile, ispirando sicurezza nel suo segmento di attività e prima della società, è importante che abbia valori basati sulla morale, l'etica e la giustizia. Dovrebbe agire con trasparenza, che gli consente di costruire la sua credibilità, e anche attrarre talenti umani che migliorano la sua capacità di agire, mirando alla sua crescita (JORGE e TOMAZ, 2018).

In quest'ottica, affinché un'organizzazione agisca con etica, buoni principi e competitività, la lotta alle frodi è un tema fondamentale, inibendo qualsiasi tipo di illecito, attraverso l'adozione di pratiche e processi a tutela dei propri valori e obiettivi, adottando un programma di conformità.

La conformità presuppone "l'adesione e il rispetto di norme e regolamenti". Secondo Antonik apud Jorge e Tomaz (2018, p. 108), conformità (chiamato anche compliance)

RC: 103918

Disponibile in: https://www.nucleodoconhecimento.com.br/economiaaziendale/vantaggi-della-conformita 
deriva dall'inglese "to comply", che tradotto significa "regole, istruzioni, norme, linee guida o semplicemente rispondere a un comando".

Coelho e Santos Júnior (2021, p. 8) sottolineato che "l'obiettivo degli standard di conformità è quello di concentrarsi sul risultato da raggiungere, cioè evitare i rischi derivanti dal commettere comportamenti personali o organizzativi considerati illegali o incoerenti con i principi, le missioni, la visione o gli obiettivi di un'azienda".

Nel corso del tempo, tra il 1907 e il 1977, sono emerse leggi applicabili anche negli Stati Uniti con portata internazionale, per controllare e punire cittadini, aziende e agenzie governative che si impegnano in azioni di corruzione.

Secondo Nascimento (2018), conformità è stata creata negli Stati Uniti, intorno al 1970, elevata come istituto legale dall'emanazione della legge pionieristica e lotta alla corruzione o - Foreign Corrupt Practices Act (FCPA). Tale legislazione è stata sviluppata e implementata dopo il caso Watergate, che è culminato nelle dimissioni dell'allora presidente degli Stati Uniti, Richard Nixon.

Furtado e Rocha (2015, apud MICHELOTTI, 2020, p. 7-8) riferiscono che nel periodo precedente alla legge FCPA, le pratiche del capitalismo nell'apertura di nuovi mercati includono il pagamento di tangenti, "considerato un passo necessario e naturale nell'ambiente aziendale. C'era la più chiara possibilità che la tangente fosse registrata come spesa nel bilancio. E la corruzione per ottenere contratti o concessioni in questi paesi faceva parte delle regole del gioco".

Poiché la corruzione è un problema globale e coinvolge - fin dall'inizio - governi, persone, istituzioni pubbliche e/ o private, Ubirajara Costódio Filho, citata da Nascimento (2021) afferma che è qualcosa che danneggia tutte le sfere sociali, per quanto riguarda l'ambiente, la libera concorrenza, le finanze pubbliche, il commercio internazionale, democrazia e precetti repubblicani.

Ancora sulla corruzione, Nascimento (2021, n.p.) riporta inoltre Eduardo Cambi, per evidenziare che:

RC: 103918

Disponibile in: https://www.nucleodoconhecimento.com.br/economiaaziendale/vantaggi-della-conformita 
a corrupção atrasa o desenvolvimento econômico e social. Restringe a vontade soberana do povo. Apropria a coisa pública para a realização de interesses privados. Gera promiscuidade entre o poder público e o poder econômico. Concentra renda. Ressalta privilégios e desigualdades. Impede a universalização de políticas públicas e a concretização de direitos fundamentais. Destrói a cidadania e enfraquece a democracia.

Lo scopo del Foreign Corrupt Practices Act (FCPA) era quello di punire le azioni fraudolente da parte di aziende che avrebbero offerto / pagato tangenti al servizio pubblico, con l'obiettivo di espandere le imprese in tutto il mondo. Dall'adozione delle pratiche prescritte dalla legge FCPA, tutte le organizzazioni sono state obbligate a seguire regolamenti interni ed esterni basati sulle questioni etiche e morali necessarie per i rapporti commerciali ed economici con i loro partner (NASCIMENTO, 2018).

\subsection{EMERGERE DI UNA LEGISLAZIONE PER LE PRATICHE DI CONFORMITÀ}

Secondo Nascimento (2018, p. 26), il movimento globale per combattere la corruzione e la pratica radicata della corruzione "è iniziato con il Foreign Corrupt Practices Act (FCPA), o legge americana contro le pratiche di corruzione". Questo autore chiarisce che "il grado di corruzione in un determinato paese varia a seconda del modo in cui le multinazionali agiscono lì".

Tra i tassi di corruzione verificati, Nascimento (2018, p. 26-27) riporta la percentuale di aziende in tutto il mondo in cui persistono la corruzione illecita e il riciclaggio di denaro:

23\% das empresas na União Europeia e Europa Ocidental; 66\% das empresas na América Latina; $64 \%$ das empresas na Ásia; $95 \%$ das empresas na Europa Ocidental e da Ásia Central; $84 \%$ das empresas do Oriente Médio e do Norte da África; $90 \%$ das empresas da África Subsaariana.

Questo autore (NASCIMENTO, 2018, p. 26-27) afferma che la globalizzazione economica "ha portato al crimine globale di corruzione legato al riciclaggio di denaro e alla corruzione offshore".

RC: 103918

Disponibile in: https://www.nucleodoconhecimento.com.br/economiaaziendale/vantaggi-della-conformita 
Si osserva, quindi, che la letteratura sui programmi di conformità e gestione del rischio mostra che, in un dato momento, diversi paesi e i loro governi hanno deciso di affrontare pratiche illegali - sia nelle istituzioni pubbliche che nelle organizzazioni private - che da allora sono stati costretti ad adottare misure punitive e restrittive per $\mathrm{i}$ cittadini e i paesi che commettono frodi, corruzione e riciclaggio di denaro, tra gli altri, con conseguenti trattati internazionali.

I paesi che adottano programmi di prevenzione delle frodi sono firmatari dell'Organizzazione per la cooperazione e lo sviluppo economico (OCSE), tra cui: Germania, Australia, Austria, Belgio, Canada, Cile, Corea, Danimarca, Slovenia, Spagna, Stati Uniti, Estonia, Finlandia, Francia, Grecia, Ungheria, Irlanda, Islanda, Israele, Italia, Giappone, Lettonia, Lussemburgo, Messico, Norvegia, Nuova Zelanda, Paesi Bassi, Perù, Polonia, Portogallo e Regno Unito (NASCIMENTO, 2021, s.p.).

Per chiarire come questi paesi hanno scelto di adottare le buone pratiche di governo societario, è importante portare all'emergere di istituzioni e leggi statunitensi che hanno permesso l'adozione di istituti di protezione per quanto riguarda i diversi tipi di illeciti contro le organizzazioni, causando l'emanazione di leggi per gli stessi scopi in tutto il mondo.

Nel 1907 fu creata la Conferenza dell'Aia, che è "una corte di giustizia internazionale per indagare sui conflitti internazionali". Con esso è stato sviluppato un ambito per la fondazione della Banca dei Regolamenti Internazionali (BRI) (Bank for International Settlements - BIS), con l'obiettivo di promuovere "la cooperazione tra le banche centrali per raggiungere una maggiore stabilità finanziaria". Tale obiettivo è considerato dagli specialisti come "le prime radici della Compliance" (NASCIMENTO, 2021, s.p.).

Nell'aprile del 1948, l'Organizzazione europea per la cooperazione economica (OECE) è nata in Portogallo, con l'obiettivo di cooperazione economica tra i paesi europei. La sua composizione iniziale aveva 18 paesi, da evidenziare: Austria, Belgio, Danimarca, 
Francia, Grecia, Olanda, Irlanda, Islanda, Italia, Lussemburgo, Norvegia, Portogallo, Regno Unito, Svezia, Svizzera, Turchia e Germania Ovest.

Sempre nel 1948 ful'Organizzazione degli Stati Americani (OSA) (Organization of American States - OAS), basata su quattro pilastri: "democrazia, diritti umani, sicurezza e sviluppo". (NASCIMENTO, 2021, s.p.).

Ancora Nascimento (2021, s.p.), evidenzia ciò che Sanches e Renee (2017, p. 18) difendono sull'OSA: "questa convenzione mira a promuovere e rafforzare i meccanismi necessari per aiutare a prevenire, individuare e punire la corruzione nell'esercizio delle funzioni pubbliche, nonché gli atti di corruzione specificamente legati al suo esercizio".

Nel dicembre 1960, i membri dell'OECE firmarono con gli Stati Uniti e il Canada una nuova Convenzione che creò l'Organizzazione per la cooperazione e lo sviluppo economico (OCSE), quindi sostituì l'OECE. Così, nel 1961 è nata l'Organizzazione per la cooperazione e lo sviluppo economico (OCSE) (Organisation for Economic Cooperation and Development (OECD), in successione all'Organizzazione per la cooperazione economica europea, i cui obiettivi erano di "coprire lo sviluppo economico e la stabilità finanziaria dei paesi membri" (NASCIMENTO, 2021, s.p.).

Nel 1975 è stato fondato il Comitato di Basilea per la vigilanza bancaria (BCBS) (Basel Committee on Banking Supervision - BCBS), il cui scopo era quello di "regolare e supervisionare le migliori pratiche finanziarie". Nel 1998 ha promosso l'uniformità delle norme applicabili agli istituti finanziari, con l'obiettivo di mantenere le buone pratiche di Corporate Governance (NASCIMENTO, 2021, s.p.).

Nel 1977, di fronte allo scandalo Watergate, fu sviluppata la legge americana contro le pratiche di corruzione all'estero (FCPA), essendo la 1a. legge anticorruzione, successivamente emanata nel 1988 e nel 1998. Secondo Nascimento (2021, s.p.), con lo scopo di "adottare sanzioni civili, penali e amministrative a persone fisiche e giuridiche contro pratiche corruttive, il Foreign Corrupt Practices Act (FCPA), o Legge americana contro le pratiche di corruzione" (NASCIMENTO, 2021, s.p.). 
È interessante notare che la FCPA descrive come "reato il pagamento di tangenti direttamente $\mathrm{o}$ indirettamente, pagamenti a dipendenti pubblici stranieri, partiti politici, il pagamento non registrato in modo conciso, ci devono essere libri contabili" (NASCIMENTO, 2021, s.p.).

Tali indagini sono state affrontate dalla Fondazione Getúlio Vargas (FGV) (2021, s.p.), segnalando l'emergere della Securities and Exchange Commission (SEC), che è "un'organizzazione amministrativa per supervisionare e combattere le pratiche di corruzione nelle transazioni internazionali".

La SEC "ha riscontrato pratiche di pagamenti illegali a funzionari pubblici da parte di una media di 400 società private e pubbliche statunitensi, e anche straniere". In tale dichiarazione, la SEC ha rilevato che "insieme hanno pagato più di $\$ 300$ milioni in tangenti a funzionari governativi stranieri" (FGV, 2021, s.p.).

Nel 1989 - per un'azione congiunta con l'OCSE - è stato creato il Gruppo di azione finanziaria internazionale (GAFI) (Financial Action Task Force - FATF), finalizzato al processo decisionale e alle riforme legislative e regolamentari, basate su leggi internazionali, volte a combattere il riciclaggio di denaro e il finanziamento del terrorismo. II GAFI agisce anche nella composizione dei meccanismi di valutazione periodica di altri paesi (NASCIMENTO, 2021, s.p.).

In pratica, il testo dell'OSA ha portato la Convenzione interamericana contro la corruzione, firmata nel 1996, volta a "proteggere gli interessi degli Stati Uniti in termini di terrorismo e corruzione".

Nel 2002 è stato implementato il Sarbanes Oxley Act (SOX) (Sarbanes-Oxley Act SARBOX) per promuovere una migliore "governance aziendale, monitoraggio e prevenzione delle pratiche dannose, aumentando i controlli e la trasparenza". Con l'istituzione dell'Organismo di Vigilanza dei Revisori dei Conti ha anche istituito verifiche indipendenti per $\mathrm{i}$ casi sospetti, rafforzando la "responsabilità per frode societaria o criminale, aumentando il livello di divulgazione delle informazioni 
finanziarie, aumentando le sanzioni per i reati da lavoro dipendente (NASCIMENTO, 2021, s.p).

Nel 2010 compare nel Regno Unito lo (UK Bribery Act (BA), che caratterizza quattro reati: "corruzione attiva di soggetti pubblici e privati, corruzione passiva di soggetti pubblici e privati, corruzione di agenti pubblici stranieri, fallimento aziendale nella prevenzione della corruzione" (NASCIMENTO, 2021, sp).

A loro volta Madruga e Belloto hanno sviluppato una guida con 10 pilastri che, una volta adottati, consentono l'attuazione di un efficiente Programma di Conformità (NASCIMENTO, 2018, p. 8-9):

1. Compromisso de todos os níveis da gerência e política anticorrupção claramente articulada; 2 . Código de conduta e política e procedimentos de compliance; 3. Fiscalização interna do programa, autonomia da área de compliance e recursos adequados; 4. Avaliação dos riscos da empresa; 5. Treinamentos e aconselhamento contínuo; 6 . Incentivos e medidas disciplinares; 7. Investigações (due diligence) de terceiros e de pagamentos; 8. Sistema interno de denúncias (possibilitando denúncias confidenciais) e sistema de investigações internas; 9. Melhoria contínua: testes e revisões periódicas do programa de compliance; 10. Fusões e Aquisições: Investigações (due diligence) pré-fusões e aquisições e integrações pós-aquisições e fusões.

Per quanto riguarda il Brasile, e nonostante l'emanazione della legge n. 12.846/13, il cui scopo è l'adozione di misure legali e procedure interne che le organizzazioni devono seguire attraverso l'adozione di un codice di condotta per combattere la corruzione, l'ingresso nell'OCSE non è stato ancora reso ufficiale da questo organismo, in considerazione dei costanti casi di corruzione, frode e riciclaggio di denaro che sorgono qui (DURÃES e RIBEIRO, 2020).

\subsection{LE PRATICHE FRAUDOLENTE E LE PERDITE CAUSATE DA TERZI ALLE ORGANIZZAZIONI}

Quando le persone legate a una determinata organizzazione adottano comportamenti che danneggiano l'immagine dell'azienda, le loro azioni sbagliate causano ripercussioni negative su di essa, così come verso l'intera società. Ci sono molti casi

RC: 103918

Disponibile in: https://www.nucleodoconhecimento.com.br/economiaaziendale/vantaggi-della-conformita 
di condotta riprovevole che scuotono non solo l'immagine, ma portano anche perdite, multe e talvolta bancarotta.

Per quanto riguarda i diversi tipi di frode allinterno di organizzazioni e istituzioni governative, come la fuga di informazioni riservate, la corruzione passiva / attiva, il riciclaggio di denaro, tra gli altri, Gonsales (2016) afferma che tutti gli studi relativi a tali frodi sostengono che in ogni azienda si verificano. Quindi, più il tuo leader crede che non stia accadendo nulla di sbagliato, più vulnerabile diventa la tua organizzazione.

In Brasile, ci sono pratiche fraudolente conosciute in tutta la società, come "falsificare la licenza di uno studente o rubare linee televisive via cavo", a cui fanno riferimento Condé et al. (2015, pag. 95); sono atti che consistono nel dimostrare la malafede di alcuni e la loro chiara intenzione di ingannare gli altri. Sfortunatamente, sono azioni praticate da molte persone, ma che nel tempo sono diventate così comuni che finiscono per essere accettate nella cultura brasiliana. Sono atti che dimostrano una mentalità e un comportamento non etici e illegali; tuttavia, sono considerati non dannosi per la società, il che è falso.

Rispetto alle azioni di cui sopra, Condé et al. (2015, p. 95) suggerisce che se pratiche non etiche, fraudolente e corrotte fossero condotte da dirigenti di alto rango di un'organizzazione, sarebbero considerate dannose e inaccettabili dalla società.

A questo proposito, Cossenzo (2015, p. 35) riporta Oliveira (2012, p. 115) per definire che "una frode finanziaria viene effettuata da atti volontari del suo agente, con la chiara intenzione di danneggiare finanziariamente gli altri". Questo autore continua spiegando che la frode può essere (i) interna - se commessa dai dipendenti contro l'organizzazione; (ii) esterno - se commesso da terzi non soci della società; (iii) misto: se le azioni fraudolente sono compiute da agenti interni ed esterni.

Secondo questa spiegazione, vale la pena evidenziare un concetto relativo alla frode finanziaria, considerato come "qualcosa inerente al business" di questo tipo di 
istituzione, classificandosi come rischi operativi relativi sia a questo segmento che "a persone, processi e tecnologia" (COSSENZO, 2015, p. 35).

Dovrebbe essere chiaro che la conformità copre tutti i tipi di frode che possono verificarsi in diversi segmenti di attività, ovvero la conformità cerca di mitigare i rischi che possono essere causati da comportamenti personali 0 organizzativi (indipendentemente dal fatto che provengano o meno dal settore finanziario ), se non agiscono secondo una condotta etica e secondo la politica aziendale.

\subsection{EVENTI DANNOSI PER LA REPUTAZIONE DELLE SOCIETÀ STATUNITENSI}

Secondo Haussen (2021, p. 9), "la reputazione di un'organizzazione può essere fortemente scossa da comportamenti disconnessi derivanti dalla mancata attuazione di un programma di conformità", come dimostrato dai casi di corruzione che si sono verificati negli Stati Uniti, che si sono stupiti nei media nel corso degli anni, incluso il caso Watergate - che ha portato all'emanazione del FCPA Act, oltre ad altri menzionati in questo articolo.

Tra i numerosi casi di azioni dannose per l'immagine delle grandi aziende statunitensi, compromettendone la credibilità, ne verranno evidenziati qui alcuni che hanno attirato molta attenzione nei media, come dimostrano alcuni rapporti localizzati nei media elettronici. Si tratta di storie che riportano come la mancata implementazione di un programma di conformità per guidare processi e persone all'interno di un'organizzazione finisca per permettere ad alcuni dipendenti di offuscare l'immagine e la reputazione di aziende conosciute a livello globale, con azioni riprovevoli.

Il primo rapporto di Vaz (2012) pubblicato da Rivista Exame porta un caso che si è verificato nel gigante Apple, il cui testo completo è presentato qui.

A Empresa Apple foi acusada pelo Senado nos Estados Unidos de usar filiais no exterior para evitar o pagamento de bilhões de dólares em impostos. "A Apple não apenas transferiu seus lucros para um paraíso fiscal estrangeiro,

RC: 103918

Disponibile in: https://www.nucleodoconhecimento.com.br/economiaaziendale/vantaggi-della-conformita 
mas criou um 'Santo Graal' de evasão fiscal ao montar entidades no exterior para driblar o fisco", disse o senador democrata Carl Levin.

Uma reportagem do new York Times revelou como a Apple tem feito para driblar bilhões de dólares em impostos nas suas operações nos Estados e em outros países. Segundo Martin A. Sullivan, um ex-economista do Tesouro americano, sem as táticas utilizadas para a gestão dos impostos, a empresa liderada por Tim Cook teria deixado pelo menos mais 2,4 bilhões de dólares em taxas federais nos EUA no ano passado. A empresa declarou ter pagado 3,3 bilhões de dólares em impostos ao redor do mundo em 2011 sobre os lucros de 34,2 bilhões de dólares, uma taxa de 9,8\%. A companhia declara que $30 \%$ dos lucros vem dos Estados, caso o percentual pulasse para $50 \%$, os pagamentos saltariam em 2,4 bilhões de dólares. "É razoável esperar que $70 \%$ dos lucros viriam dos Estados Unidos”, calcula.

Figura 1 - Simbolo Apple

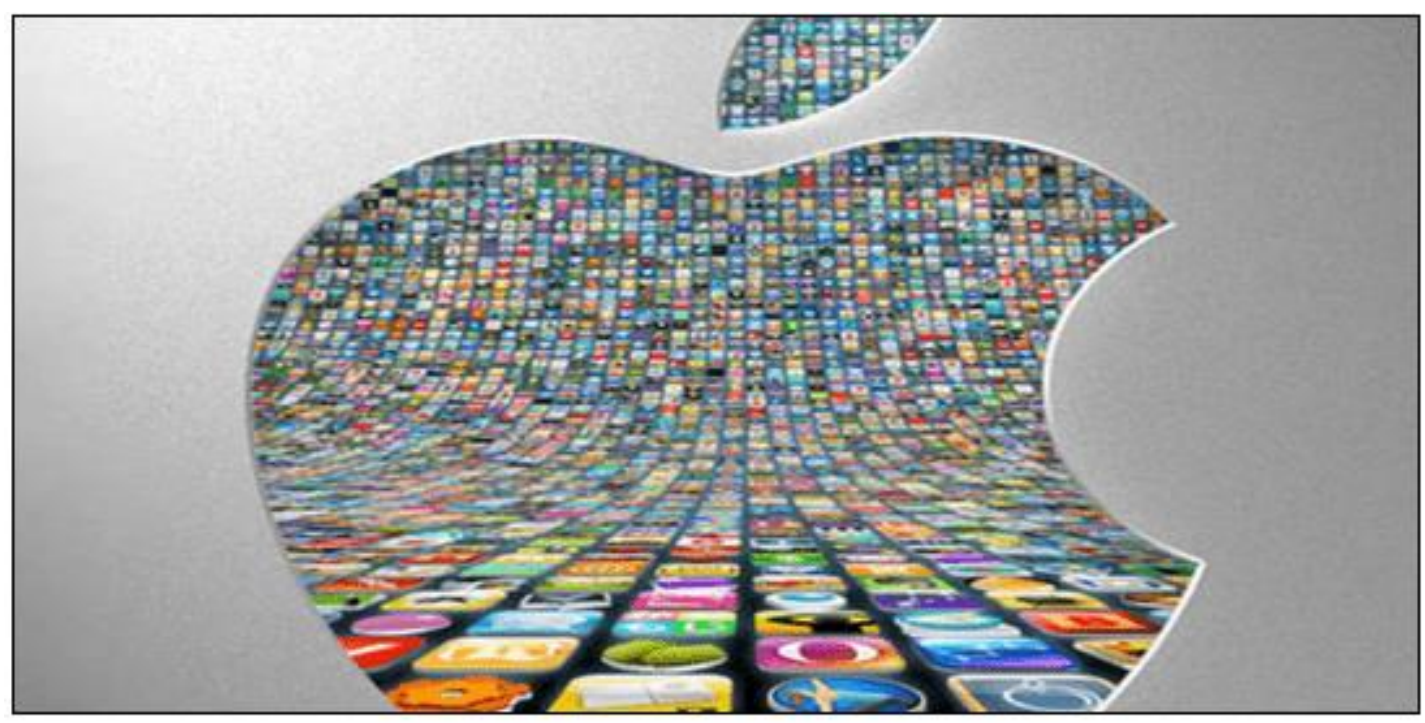

Fonte: Vaz (2012)

Per quanto riguarda le possibili cause che possono compromettere la credibilità delle organizzazioni, Souza; Maciel-Lima e Lupi (2018, p. 13) fanno riferimento a Gonsales (2016), per spiegare che:

o valor de uma empresa está ligado à sua reputação e no caso de uma empresa ser citada em jornais e meios de comunicação, ainda que seja um ato ilícito isolado, acometido por um funcionário, a empresa sofrerá abalo a reputação e passa a ser conhecida no mercado como uma empresa corrupta.

RC: 103918

Disponibile in: https://www.nucleodoconhecimento.com.br/economiaaziendale/vantaggi-della-conformita 
Il secondo rapporto si riferisce a due diverse situazioni, che si sono verificate in due diverse unità nei ristoranti nordamericani del Reti Taco Bell, fast food ispirato alla cucina messicana, che segue il modello McDonalds.

Il primo è nel rapporto di Melo (2014) pubblicato da Rivista Exame, il cui testo segue di seguito.

Publicar uma foto de si mesmo fazendo xixi em um prato do Taco Bell, nos Estados Unidos, não custou ao atendente Cameron Jankowski apenas o emprego. Indignados, hackers divulgaram os dados pessoais do "brincalhão" na internet, segundo o Huffington Post. Em 2012, Jankowski postou imagem no Twitter, mas disse que não chegou a servir a comida no restaurante. Ele chegou a deletar sua conta, mas acabou sem seu emprego na rede.

Figura 2 - Pannello elettronico Taco Bell

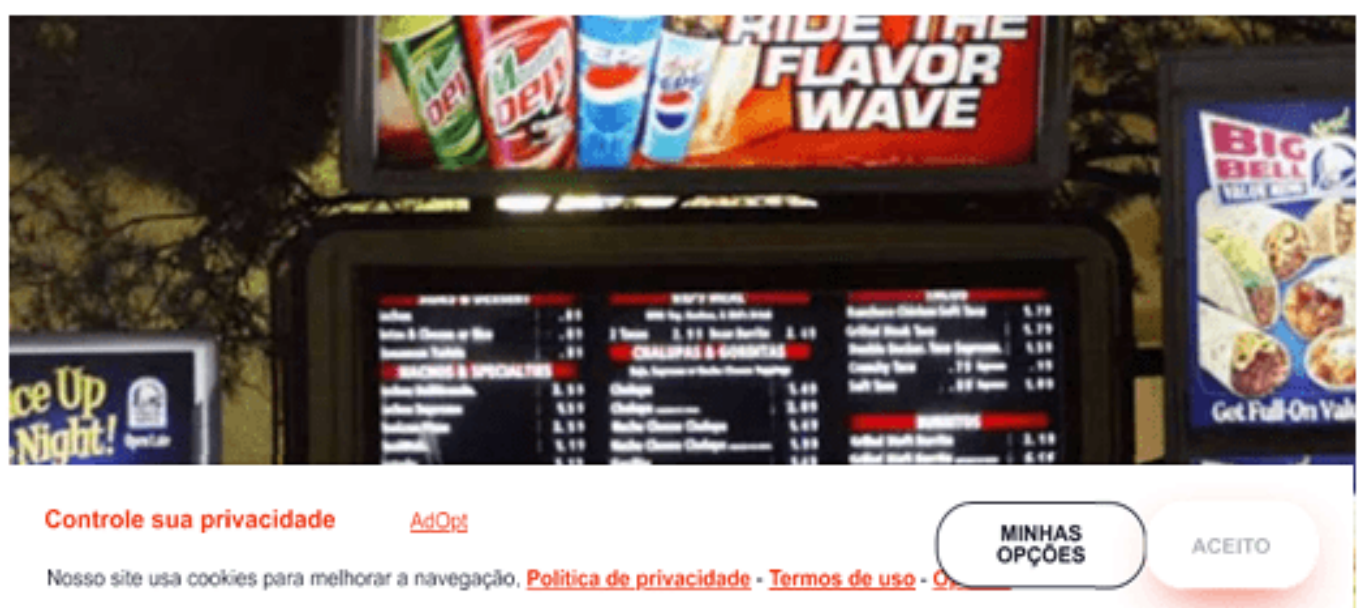

Fonte: Melo (2014)

Nel 2013, la stessa rete avrebbe attraversato un altro momento che ha causato danni alla sua credibilità. II rapporto è tratto da Rivista Veja On Line (2014), il cui testo segue per intero.

Uma imagem de um funcionário da rede de comida mexicana Taco Bell lambendo uma pilha de tortilhas foi postada na página da empresa no Facebook. A foto, de autoria do usuário Jj O'Brien Nolan, é seguida da legenda "isto com certeza diz muito sobre seus empregados, o tratamento da comida e o que eles postam na internet.

RC: 103918

Disponibile in: https://www.nucleodoconhecimento.com.br/economiaaziendale/vantaggi-della-conformita 
Apesar de inicialmente acreditar que a foto fosse uma brincadeira, a empresa demitiu o empregado. Além disso, em resposta, a Taco Bell divulgou um comunicado oficial, além de comentar a postagem de Nolan no Facebook. A empresa se defendeu afirmando que todas as unidades têm rígidos procedimentos de manuseamento e tolerância zero para violação dos alimentos.

Figura 3 - Impiegato Taco Bell che lecca un mucchio di tortillas

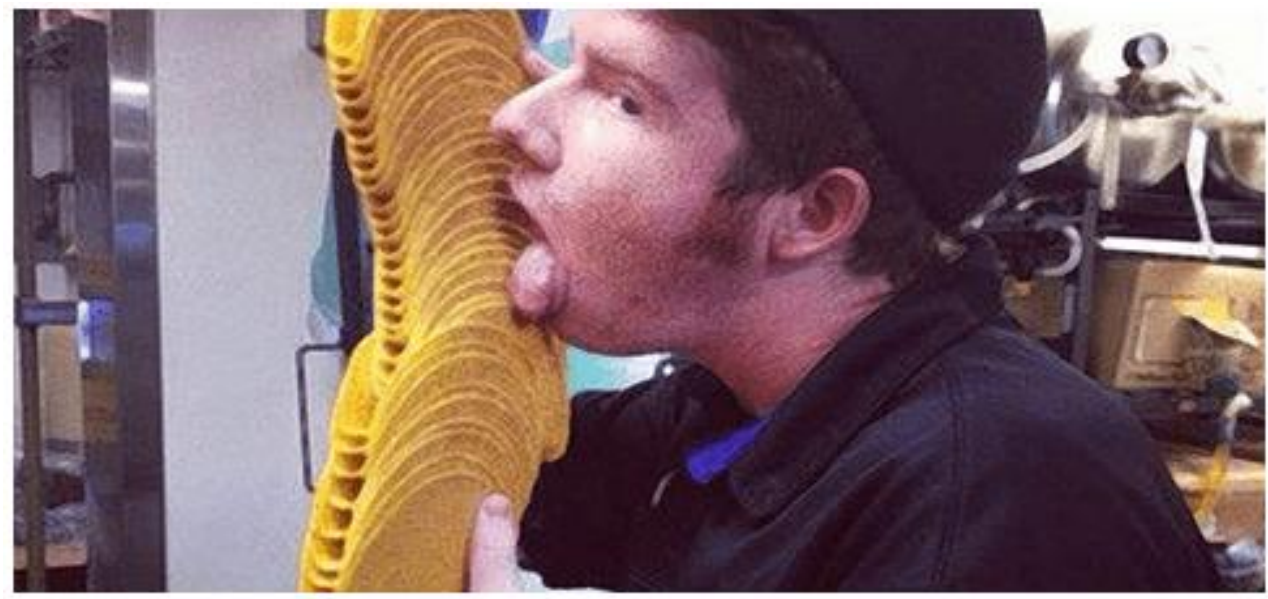

Fonte: Veja online (2014)

Un terzo caso da segnalare è stato svelato in un articolo di Melo (2014, edito da Rivista Exame, avvenuto in uno dei ristoranti di Reti KFC, in Nord America, il cui testo originale è qui riprodotto.

Questo è stato il caso in cui un ex dipendente della catena di ristoranti KFC negli Stati Uniti ha pubblicato una foto su Facebook mentre stava letteralmente leccando il purè di patate. Lei e la sua amica fotografa sono state licenziate. La rete ha dovuto giustificarsi dicendo che il cibo non era servito.

RC: 103918

Disponibile in: https://www.nucleodoconhecimento.com.br/economiaaziendale/vantaggi-della-conformita 
Figura 4 - Dipendente KFC che lecca purè di patate
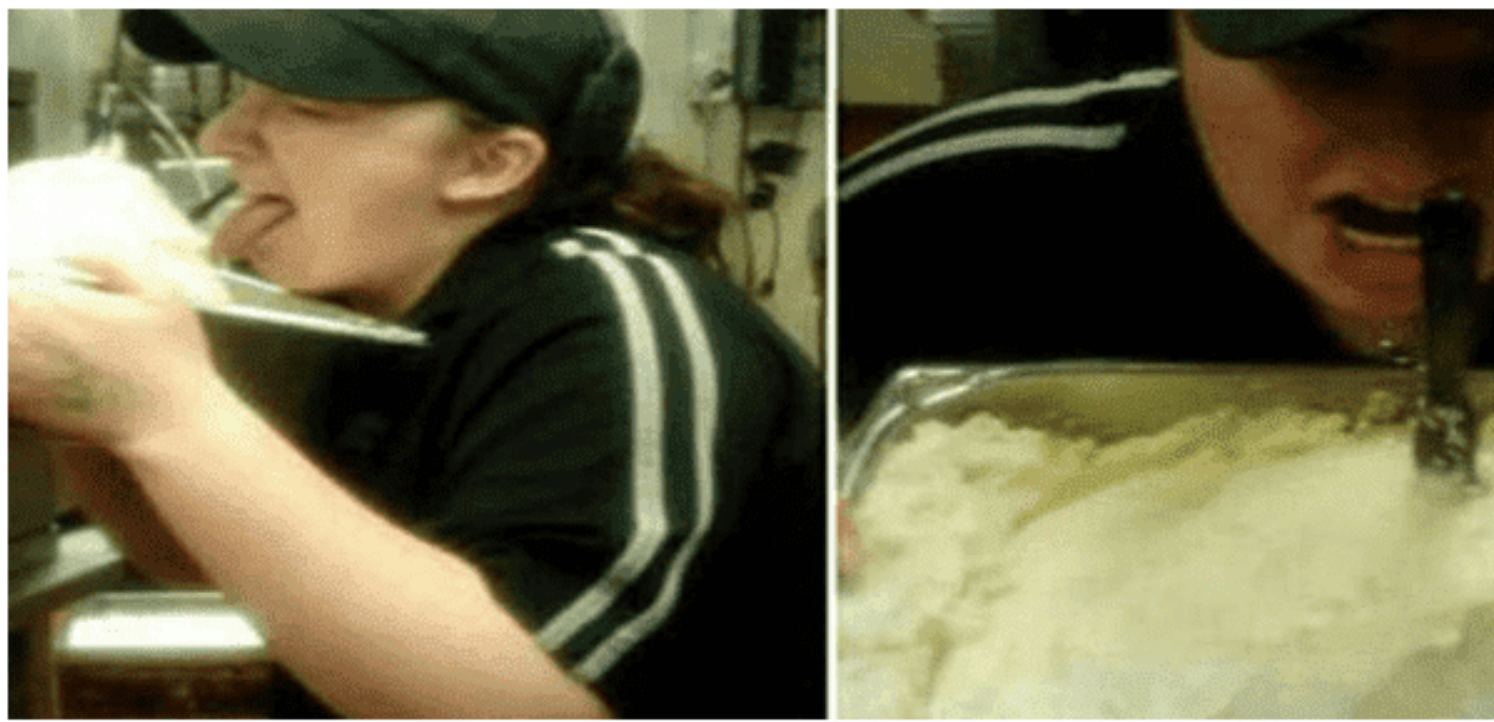

Fonte: Melo (2014)

I casi di condotta non etica presentati riflettono l'importanza nell'adozione di pratiche di conformità e Gestione del rischio. Dal punto di vista di Coelho e Santos Júnior (2021, p. 26), "non è sufficiente che l'azienda abbia condizioni finanziarie per investire in pubblicità e marketing per guidare il mercato".

Ciò che viene verificato, in pratica, è che le organizzazioni devono tenersi aggiornate in modo permanente, attente alle nuove pratiche di marketing, oltre ad adottare strategie che consentano loro di essere competitive nel loro segmento di attività, offrendo servizi migliori, prezzi competitivi e dando opzioni di scelta ai loro consumatori target.

In questo senso, Coelho e Santos Júnior (2021, p. 27) sostengono che "tra i fattori che influenzano il livello di competitività aziendale ci sono: capacità di innovazione, prodotti e servizi di qualità, differenziali commerciali, capacità di produzione e consegna, portata geografica, azioni di marketing; tra gli altri." 


\section{RISCHI DI CONFORMITÀ}

Non adottando programmi di prevenzione e mitigazione delle frodi nei loro processi organizzativi, le aziende sono soggette al verificarsi di diversi eventi illeciti, poiché "la frode si verifica in tutte le società". Inoltre, sono vulnerabili a due tipi di rischi molto dannosi: lo scuotimento della loro reputazione e le punizioni previste dalla nuova legge anticorruzione (GONSALES, 2016).

Il termine "Rischio di conformità" si riferisce ai rischi a cui le aziende sono soggette da sanzioni normative, perdite finanziarie e/o perdite causate alla loro immagine, rischi derivanti dal mancato rispetto di regolamenti, codici di condotta e buona condotta (COELHO e SANTOS JR, 2021, p. 8).

Secondo Gonsales (2016) il 43\% delle aziende brasiliane non ha ancora adottato programmi e politiche di conformità per i propri processi e la propria protezione, ma che presto tutti saranno costretti a farlo, mirando a proteggere la propria reputazione, poiché "il valore di un'azienda è legato alla sua reputazione.

È una realtà che ha trasformato l'ambiente commerciale e industriale nazionale, derivante dalle indagini di Mensalão e Operação Lava Jato e successive, poiché lo smantellamento della frode finanziaria e la corruzione comprovata e il riciclaggio di denaro dimostrano la necessità di un nuovo ambiente imprenditoriale, che richiede trasparenza e integrità nella conduzione degli affari. Le punizioni e gli arresti di dirigenti di alto livello e funzionari governativi di alto rango sono avvenuti alla luce della nuova legge anticorruzione istituita nel paese (GONSALES, 2016).

I dati ricercati da PwC Brazil (2020, p. 4) mostrano che il rischio di conformità relativo alle frodi e ai crimini economici più comuni si verifica con le organizzazioni sia a livello nazionale che internazionale. Secondo questa ricerca, è stato riscontrato che in Brasile sono tra i tre tipi più comuni di frode: (1) corruzione e concussione, (2) frode contabile e (3) frode commessa dal consumatore. Poiché l'indagine è stata condotta nel 2020, è emerso che negli ultimi due anni (tra il 2018 e il 2019), le frodi più comuni apparse 
nelle prime posizioni sono state: (a) furti di beni, (b) frodi negli acquisti e (c) tangenti e corruzione.

A loro volta, secondo PwC (2020, p. 4) i dati comparativi della classifica delle frodi che appaiono nelle prime posizioni in tutto il mondo erano: (1) frodi commesse dai consumatori, (2) crimini informatici e (3) furti di beni. Poiché l'indagine è stata condotta nel 2020, è stato dimostrato che negli ultimi due anni (tra il 2018 e il 2019), le frodi più comuni apparse nelle prime posizioni sono state: (a) frode commessa dal consumatore, (b) frode contabile (c) concorrenza sleale, (d) frode delle risorse umane e (e) corruzione e concussione.

La Figura 5 mostra i dati nelle prospettive interne ed esterne. 
Figura 5 - I tipi più comuni di reato

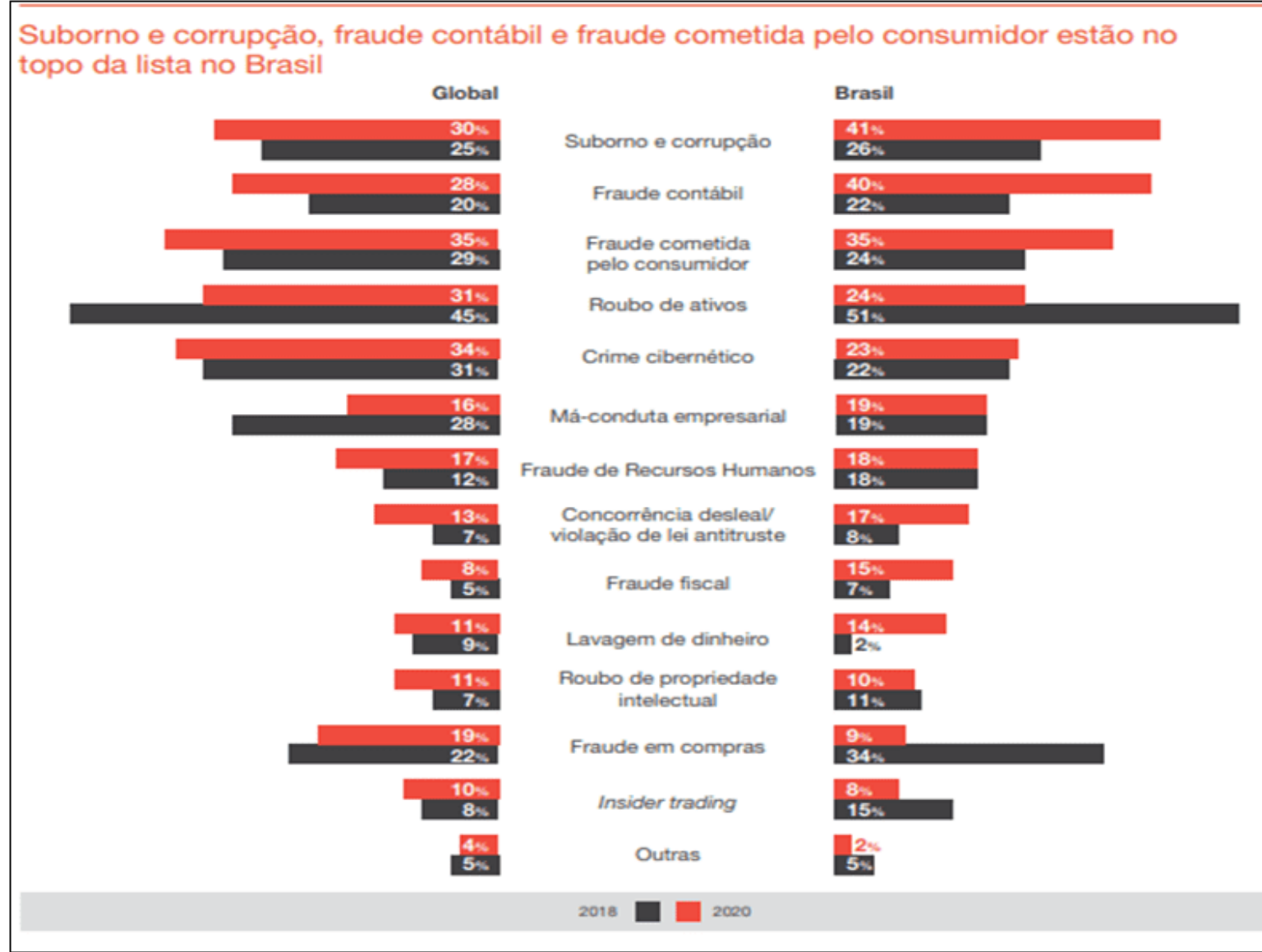

Fonte: PwC Brasil (2020, p. 5)

Sono crimini economici che, oltre a danneggiare i beni delle organizzazioni, compromettono la loro reputazione e immagine, essendo possibili fino a raggiungere la bancarotta. È in quest'ottica che i programmi di conformità consentono di evitare rischi, comportamenti illeciti e non etici, dall'attuazione di uno specifico programma che contenga chiaramente il Codice Etico e la Politica Interna dell'organizzazione da seguire, conferendo "crescita, investimento, credibilità, sicurezza e protezione" (PwC, 2020).

In questo senso, Oliveira (2012 apud COSSENZO, 2015, p. 35) afferma che la prevenzione dovrebbe essere fondamentalmente composta dall'impegno che le aziende devono stabilire internamente, relativo alla condotta etica per decisioni e

RC: 103918

Disponibile in: https://www.nucleodoconhecimento.com.br/economiaaziendale/vantaggi-della-conformita 
comportamenti, permeando i loro processi aziendali e operativi, e può anche adottare pratiche preventive, relative alla storia di dipendenti, fornitori e clienti a, rafforzare una cultura antifrode interna ed esterna.

Tra i rischi della Compliance, vale la pena menzionare la Teoria del triangolo della frode, che evidenzia tre aspetti che aumentano le possibilità di atti illegali contro le organizzazioni, sono: pressione, opportunità e razionalizzazione, come spiegato da Condé, Almeida e Quintal (2015). Questo triangolo può essere visualizzato dalla Figura 6.

Figura 6 - Triangolo delle frodi

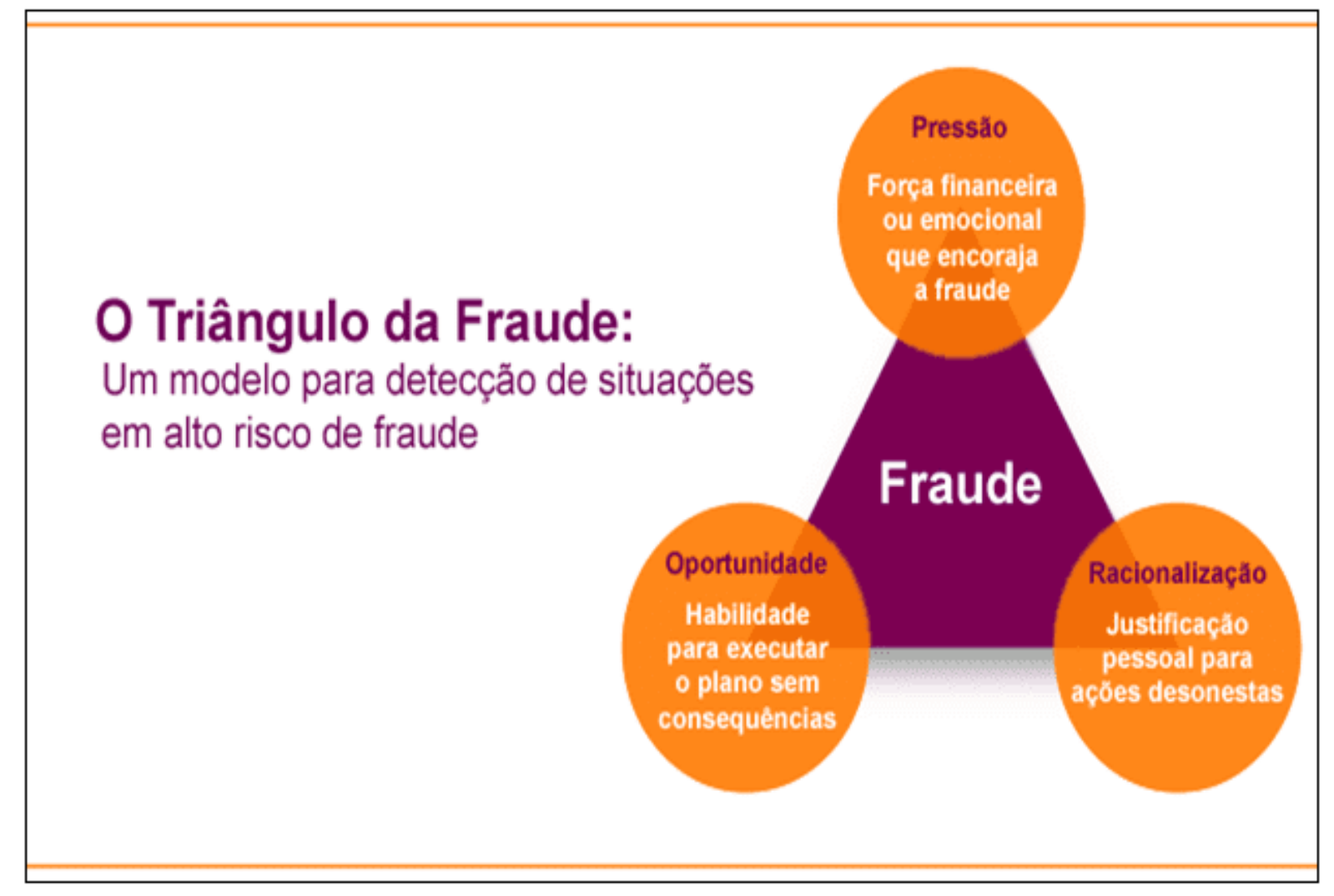

Fonte: Lustosa (2016)

Ancora Condé et al. (2015) definire ciascuno degli elementi che compongono il triangolo delle frodi:

RC: 103918

Disponibile in: https://www.nucleodoconhecimento.com.br/economiaaziendale/vantaggi-della-conformita 
a "pressão" geralmente está ligada ao motivo que leva ao crime, uma necessidade econômica "estressante" da empresa ou do funcionário. A "oportunidade" de cometer o ato ilícito, com baixo risco de ser descoberto ou nenhum. A "Racionalização" de uma autojustificação pelo cometimento do ilícito que para o fraudador seria "aceitável".

In considerazione di questo scenario, si nota la necessità di una mappatura dei rischi organizzativi, attraverso la prevenzione e l'identificazione dei rischi, tra cui il mancato rispetto della legislazione e del codice di condotta organizzativo, il rischio operativo, il rischio di immagine dovuto a negligenza o mancanza di comportamenti appropriati, o anche rischi esterni come la domanda politica del paese, sconvolgimenti tecnologici, salute e instabilità economica, aspetti che possono interessare anche le organizzazioni.

In questo senso, Cossenzo (2015) spiega che per gestire le frodi sono necessarie strategie che le identifichino al momento attuale, oltre ad altre, che consentano la loro prevenzione. Quando vengono identificati i rischi di frode, è anche necessario identificare come si verificano e le persone che li praticano, e per questo, è essenziale che le aziende condividano le informazioni tra loro.

Lo stesso autore sottolinea l'esistenza delle Commissioni per la lotta contro le frodi della Federazione brasiliana delle banche (FEBRABAN), la Commissione di base per l'incoerenza dell'Associazione nazionale degli istituti di credito, finanziamento e investimento (ACREFI) e l'accordo di cooperazione firmato nel 2009 tra la polizia federale e Febraban, per quanto riguarda i controlli elettronici con informazioni sui casi che si sono verificati (COSSENZO, 2015, p. 35).

Per quanto riguarda le frodi esterne, Oliveira (2012, p. 73-4) citata da Cossenzo (2015, p. 36) insegna che ci sono tre fasi preventive: "prevenzione, individuazione e reazione", tutte in una fase globale, chiamata "continua". Tali fasi possono essere osservate nel grafico 1. 
Tabella 1 - Fasi e categorie della prevenzione delle frodi esterne

\begin{tabular}{|l|l|}
\hline Fase & Categorie correlate \\
\hline Continuo & Responsabilità \\
\hline & Sinergia \\
\hline Prevenzione & Miglioramento \\
\hline Scoperta & $\begin{array}{l}\text { Istituzione } \\
\text { Identificazione }\end{array}$ \\
\hline Reazione & Manutenzione \\
\hline Ricerca \\
\hline
\end{tabular}

Fonte: Preparato dall'autore (2021)

Per prevenire tali eventi, dovrebbero essere adottate azioni per sensibilizzare le persone coinvolte e dovrebbero essere definiti e attuati controlli, inibendo nuovi eventi; per la loro individuazione dovrebbero essere adottati metodi di indagine e conferma dei sospetti circa il verificarsi o meno di frode; e per reagire, che le azioni sono stabilite dall'azienda, quando viene rilevata una frode.

\subsection{SOLUZIONI DI ENTERPRISE GOVERNANCE, RISK MANAGEMENT AND COMPLIANCE (EGRC)}

Tra i vantaggi che l'implementazione di un programma di conformità e gestione del rischio promuove all'interno delle organizzazioni vi sono la protezione contro le frodi e gli illeciti, promuovendo la mitigazione di tali rischi.

Affinché le misure di sicurezza siano efficacemente efficaci, attraverso una realtà sempre più tecnologica, è necessario che vengano sviluppati e implementati strumenti e soluzioni per combattere le frodi, consentendo una maggiore conformità con le pratiche necessarie per il successo delle organizzazioni, come spiegato da Grand View Research (2014). Sono misure inerenti all'Governance delle tecnologie

RC: 103918

Disponibile in: https://www.nucleodoconhecimento.com.br/economiaaziendale/vantaggi-della-conformita 
dell'informazione, nonché all'Internet of Things (IOT), o Internet of Things, che a loro volta sono inerenti alla Corporate Governance (CHAVES, 2014).

La Corporate Governance consiste in un "sistema di rapporti tra azionisti, sindaci indipendenti, dirigenti societari e amministratori guidato dal consiglio di amministrazione" (LODI, 2000, citato da CHAVES, 2014, pag. 21). A sua volta, I'IT Governance, attraverso "big data e analytics, loT, apprendimento automatico e Al e social media, comporta la condivisione di informazioni personali, rendendo gli utenti e le aziende più suscettibili agli hacker" (GRAND VIEW RESEARCH, 2014).

Le risorse portate dall'loT e dalla digitalizzazione dei processi aziendali sono cresciute rapidamente, consentendo interazioni con i dati e fornendo un maggiore controllo delle informazioni da parte del management delle aziende. In pratica, l'obiettivo è quello di affidarsi all'Intelligenza Artificiale (IA) per gestire gli obblighi e le report esterni, che consentono una maggiore corporate governance, gestione dei rischi e della conformità - EGRC (GRAND VIEW RESEARCH, 2014).

Secondo Chaves (2014, p.22), "per I'Istituto brasiliano di Corporate Governance (IBGC, 2006), I'IT Governance è un braccio della Corporate Governance", che consente l'elaborazione e l'attuazione di "normative ad alto impatto nell'area IT delle aziende, che richiedono maggiore impegno ed efficacia nella pianificazione, nei controlli, nel monitoraggio e nei processi di sicurezza relativi alle strategie aziendali". Ancora Chaves (2014, p. 22) riporta una definizione data dall'Information Technology Governance Institute (ITGI, 2012), per quanto riguarda gli obiettivi dell'IT Governance:

compreender os assuntos e a importância estratégica de TI, assegurar que a empresa possa sustentar suas operações e implementar as estratégias requeridas para expandir as suas atividades no futuro. As práticas de Governança de TI possibilitam que: sejam atendidas as expectativas dos clientes de TI, que a performance de TI seja medida, os seus recursos possam ser gerenciáveis e os riscos do negócio mitigados.

Nel suo turno. Siqueira (2019) fa riferimento alla Indagine sui rischi per la sicurezza informatica e dati, preparata da Marsh e McLennan nel 2018, mostrando che solo il

RC: 103918

Disponibile in: https://www.nucleodoconhecimento.com.br/economiaaziendale/vantaggi-della-conformita 
$18 \%$ delle piccole imprese avrebbe sviluppato un qualche tipo di piano di risposta agli incidenti informatici.

In quest'ottica, la creazione e l'implementazione di soluzioni EGRC offrono la possibilità di superare le sfide esistenti - come le frodi elettroniche e la clonazione, ad esempio, in un universo di business "iperconnesso", che può essere ottenuto attraverso software specifici. Rappresenta inoltre uno strumento che collabora in modo sostanziale nella protezione dei dati e nella proprietà intellettuale (PI), oltre a migliorare la gestione del lavoro a distanza, un modello ibrido così comune durante tutta la pandemia da Covid 19 (GRAND VIEW RESEARCH, 2014).

\section{VANTAGGI DI UN PROGRAMMA DI CONFORMITÀ E GESTIONE DEL RISCHIO}

Secondo il portale 3Mind Legale (2021), tra i concetti principali di conformità e gestione del rischio c'è la generazione di valore aziendale, che mira a consentire la sopravvivenza e la longevità delle organizzazioni. Tra i pericoli e gli impatti finanziari dell'assenza di un programma che promuova il buon governo ci sono: "assenza di linee guida normative; mancanza di adeguati strumenti di prevenzione; disallineamenti alla normativa applicabile; sistema informativo senza operazioni strutturate; gestione dei processi fallimentari".

Poiché le istituzioni pubbliche e private dovrebbero guidare la loro gestione nei programmi di Corporate Governance, Klen (2009, apud SENNO et al., 2019, p. 225) definisce che "la corporate governance è il sistema attraverso il quale le società commerciali sono gestite e controllate".

In questo senso, ancora Senno et al. (2019) spiegare che le pratiche di governo societario devono convergere per garantire meccanismi che consentano il ritorno finanziario degli investimenti effettuati, attraverso l'adozione di norme giuridiche e la responsabilità nei confronti di tutte le parti interessate.

RC: 103918

Disponibile in: https://www.nucleodoconhecimento.com.br/economiaaziendale/vantaggi-della-conformita 
A sua volta, Azevedo et al. (2017, p. 181) spiegano che governance si riferisce al governo, mentre il governo societario si riferisce al sistema con cui organi e poteri sono organizzati all'interno di un'azienda", che deve essere supportato da quattro principi: trasparenza, integrità o equità, responsabilità e rispetto delle leggi.

Queste spiegazioni mirano a collaborare per comprendere meglio le buone pratiche di governance, che, secondo KPMG (2021), richiedono alcuni elementi specifici per comporre un efficiente programma di conformità normativa, che sono:

1. Politica e programma di conformità;

2. Messaggio di leadership e cultura della compliance;

3. Strutturazione del conformità officer;

4. Comunicazione e formazione della politica e del programma di conformità;

5. Monitoraggio, auditing e valutazione dell'efficacia della politica e del programma etico e di conformità;

6. Incentivi per prestazioni e azioni disciplinari;

7. Ricerca e prevenzione.

In questa prospettiva, si segnala che un programma di conformità implica standard anti-corruzione, anti-corruzione e di conformità pubblica. Per maggiori dettagli, sono elencati i vantaggi forniti da Compliance,secondo il portale 3Mind legale (2021):

1. Attrarre investitori e investimenti;

2. Aumentare la governance in azienda;

3. Consolidare la cultura organizzativa;

4. Correggere efficacemente le non conformità;

5. Guadagnare credibilità;

6. Ottenere un vantaggio competitivo rispetto alla concorrenza;

7. Identificare i rischi e prevenire i problemi;

8. Migliorare l'efficienza e la qualità dei prodotti e dei servizi offerti;

9. Sostenibilità. 
A sua volta, Caovilla (2017) descrive alcuni vantaggi legati all'adozione della Compliance da parte delle organizzazioni (i nostri grifoni):

1. Nuove opportunità di business e vantaggio competitivo: le aziende che operano in conformità con le leggi e le buone pratiche cercano di relazionarsi con coppie uguali, oltre ad aumentare le loro opportunità in relazione a coloro che non utilizzano programmi di conformità;

2. Nuovi investimenti: le organizzazioni con bassi tassi di coinvolgimento in scandali illeciti e sociali, dimostrando solidità sono più attraenti per gli investitori interni ed esterni;

3. Mitigazione del rischio: le aziende che adottano un programma di conformità, oltre a identificare possibili rischi per la propria attività, sono in grado di sviluppare e attuare azioni per mitigare eventuali guasti e rischi;

4. Correzione delle non conformità: oltre alle azioni preventive per evitare possibili non conformità, sono le azioni per correggere le non conformità individuate nei processi interni di un'organizzazione. Tali azioni necessarie per preservare l'immagine e la reputazione di un'azienda possono essere: correzione del piano d'azione in corso; formazione per i dipendenti; revisione delle politiche, delle procedure, dei comportamenti, dei dipendenti e dei fornitori e dei fornitori di servizi;

5. Conformità come consapevolezza delle persone: una pratica che consente la mitigazione delle frodi, poiché se i dipendenti e gli altri attori sono formati alla conformità , sono in grado di vedere le non conformità all'interno e all'esterno dell'azienda, cioè in concorrenza, nei fornitori, nei fornitori di servizi;

6. La conformità come limitazione di responsabilità: le conoscenze acquisite nella formazione e nelle pratiche etiche legate alla conformità consentono la selezione di migliori partner commerciali, oltre a tutelare la reputazione di un'azienda. Poiché le buone pratiche permeano le routine aziendali di un'organizzazione, eventuali non conformità possono portare alla riduzione delle sanzioni che possono essere applicate ad essa; 
7. La conformità come sostenibilità aziendale: l'adozione e il mantenimento di pratiche etiche e trasparenti consentono alle organizzazioni di rimanere a lungo termine.

\section{CONCLUSIONE}

Attraverso l'analisi dei riferimenti teorici selezionati per lo sviluppo di questo articolo, è stato possibile osservare che un programma di Conformità e Gestione del rischio ha grande importanza per la corretta gestione di processi, pratiche e routine all'interno delle organizzazioni, coinvolgendo tutti i loro settori e personale.

Ritornando al punto di redini; in che modo i vantaggi offerti dal programma Conformità e Gestione del rischio collaborano per ridurre i rischi di frode, criminalità illecita e corruzione all'interno di organizzazioni e istituzioni governative? Concludiamo che, poiché la letteratura dimostra che ci sono frodi in tutte le organizzazioni, senza distinzione per il segmento di attività o le sue dimensioni, il beneficio che la conformità conferisce alle aziende è proprio nella protezione che offre, mitigando i diversi tipi di rischi e illeciti, anche evitando sanzioni legali.

Sulla base delle leggi stabilite, dalla Legge pionieristica per combattere la corruzione FCPA (Foreign Corrupt Practices Act), i paesi firmatari dell'OCSE, elencati in questo articolo, hanno adottato iniziative simili per promuovere la mitigazione di frodi, corruzione e azioni non etiche nei loro governi e organizzazioni, fortemente sostenuto dalle restrizioni e dalle punizioni imposte dalle leggi.

Inoltre, è emerso lo sviluppo di nuovi strumenti tecnologici, supportati dall'Governance delle tecnologie dell'informazione, a supporto della Governo d'impresa, promuovendo cambiamenti nella gestione delle organizzazioni e dei loro processi amministrativi.

Tale gestione è qualcosa che dovrebbe essere basata su strumenti moderni, non essendo limitata ai processi stessi, ma che dovrebbe consentire a un'organizzazione di anticipare eventi futuri, evitando malintesi. 
In questo contesto, le soluzioni EGRC - che consentono le best practice per I'TI Governance e la Corporate Governance - collaborano per la prevenzione e la mitigazione di frodi e azioni non avvertite, proteggendo la crescita delle organizzazioni e la loro immagine di mercato.

\section{RIFERIMENTI}

AZEVEDO, Mateus Miranda de; CARDOSO, Antonio Almeida; DUARTE, Jairo Gonçalves; FEDERICO, Bianca Ellen; LIMA, Marco Antonio Ferreira. O Compliance e a gestão de riscos nos processos organizacionais. Revista de Pós-Graduação Multidisciplinar, São Paulo, v. 1, n. 1, p. 179-196, mar./jun. 2017.

ISSN 2594-4800 | e-ISSN 2594-4797 | doi: 10.22287/rpgm.v111.507; acesso em 08 dez 2021.

CAOVILLA, Renato Vieira. Benefícios práticos do compliance. 10 jul 2017. Disponível em: [https://www.migalhas.com.br/depeso/261662/beneficios-praticos-docompliance]; 08 dez 2017.

CHAVES, Elisabete Cecília Januário. Resultados e tendências de Requisitos e Práticas de Governança de TI nos bancos de Varejo no Brasil. Dissertação [Mestrado em Gestão e Tecnologia em Sistemas Produtivos] apresentada ao Centro Estadual de Educação Tecnológica Paula Souza, no Programa de Mestrado Profissional em Gestão e Tecnologia em Sistemas Produtivos. São Paulo, novembro de 2014.

em:

[http://www.pos.cps.sp.gov.br/files/dissertacoes/file/143/7fc218f434af24b99f50af42e 8442903.pdf]; acesso em 12 out 2021.

COELHO, Cláudio Carneiro Bezerra Pinto; SANTOS JÚNIOR, Milton de Castro. Compliance, FGV/IDE, 2021.

CONDÉ, Robson Augusto Dainez; ALMEIDA, Carlos Otávio Ferreira de; QUINTAL, Renato Santiago. Fraude Contábil: análise empírica à luz dos pressupostos 
teóricos do triângulo da fraude e dos escândalos corporativos. Gestão \& Regionalidade - Vol. 31 - № 93 - set-dez/2015. doi: 10.13037/gr. vol31n93.2929; acesso em 28 out 2021

COSSENZO, Fábio. FRAUDE BANCÁRIA: A mitigação dos Riscos e Perdas Financeiras no Segmento de Crédito Consignado. Monografia de Pós-Graduação Lato Sensu - MBA Gestão empresarial, apresentada à Fundação Instituto de Administração - FIA. São Paulo, 2015.

DURÃES, Cintya Nishimura; RIBEIRO, Maria de Fátima. O Compliance no Brasil e a Responsabilidade Empresarial no combate à corrupção. Revista Direito em Debate: Revista do Departamento de Ciências Jurídicas e Sociais da Unijuí Editora Unijuí - Ano XXIX - n. 53 - jan./jun. 2020. Disponível em: [http://dx.doi.org/10.21527/2176-6622.2020.53.69-78]; acesso em 01 dez 2021.

ENTERPRISE GOVERNANCE, RISK \& COMPLIANCE MARKET SIZE. Share \& Trends Analysis Report By Component, By Software, By Services, By Enterprise Type, By Vertical, And Segment Forecasts, 2021 - 2028

FGV - FUNDAÇÃO GETÚLIO VARGAS. Surgimento do Compliance. 2021.

GONSALES, Alessandra. O que é compliance? Publicação da Legal, Ethics and Complaince (LEC). Vídeo publicado em 29 fev 2016. Disponível em: [https://www.youtube.com/watch?v=2BDpJ6UMXb4]; acesso em 01 dez 2021.

GRAND VIEW RESEARCH. Relatório de governança corporativa, risco e conformidade do mercado, análise de compartilhamento e tendências por componente, por software, por serviços, por tipo de empresa, por vertical e por segmento. Previsões, 2021-2028. Artigo publicado em abril de 2021. Disponível em: [https://www.grandviewresearch.com/industry-analysis/enterprise-governance-riskcompliance-egrc-market]; acesso em 20 nov 2021. 
HAUSSEN, Leonard. Compliance. Reunião On-Line (ROL), Aula Organizada pela Fundação Getúlio Vargas, Escola de Administração de Empresas de São Paulo, São Paulo, Brasil. Agosto de 2021.

JORGE, Glaucio Fiorenzano; TOMAZ, Roberto Epifânio. Compliance rins - como implantar e quais os benefícios do programa de compliance? IV Congresso Catarinense de Direito processual Civil \& Mais. 7 a 9 de novembro de 2018. Univali Eventos - Universidade Vale do Itajaí. Disponível em: [https://core.ac.uk/download/pdf/276005736.pdf]; acesso em 20 out 2021.

KPMG - Compliance analytics. 2018. Disponível em: [https://assets.kpmg/content/dam/kpmg/br/pdf/2018/08/br-compliance-analytics.pdf]; acesso em 08 dez 2021.

KPMG - Compliance regulatório. 2021. Disponível em: [https://home.kpmg/br/pt/home/servicos/consultoria/risk-consulting/internal-auditrisk/compliance-regulatory.html]; acesso em 08 dez 2021.

MELO, Luísa. 8 crises empresariais causadas por funcionários brincalhões. Matéria publicada em 14 mar 2014. Disponível em: [https://exame.com/negocios/8crises-empresariais-causadas-por-funcionarios-brincalhoes/]; acesso em 20 out 2021.

MICHELOTTI, Andriws Loreto. O crime de lavagem de capitais e os desafios empresariais diante da implementação dos programas de criminal Compliance. Trabalho de Conclusão de Curso [Bacharelado em Direito] apresentado à Faculdade Antonio Meneghetti (AMF). Restinga Seca (RS), 2020. Disponível em: [http://repositorio.faculdadeam.edu.br/xmlui/bitstream/handle/123456789/673/TCC_D IR_ANDRIWS_MICHELOTTI_AMF_2020.pdf?sequence=1\&isAllowed=y]; acesso em 07 dez 2021.

NASCIMENTO, Juliana Oliveira. A Função Sociald da Empresa e a Efetividade Prática da Conduta de Integridade: o Compliance Vivo. Dissertação [mestrado em Direitos Fundamentais e Democracia] apresentada ao Centro Universitário Autônomo 
do Brasil. Curitiba, 2018. Disponível em: [https://www.unibrasil.com.br/wpcontent/uploads/2019/07/Dissertação-JULIANA-OLIVEIRA-NASCIMENTO.pdf]; acesso em 29 ago 2021.

NASCIMENTO, Débora Minuncio. Evolução histórica e legislações acerca do Compliance. Artigo publicado em 07 nov 2021. Disponível em: [https://advocaciadeboramn.jusbrasil.com.br/artigos/700763578/evolucao-historica-elegislacoes-acerca-do-compliance]; acesso em 20 out 2021.

\section{OECE - ORGANIZAÇÃO PARA A COOPERAÇÃO E DESENVOLVIMENTO ECONÔMICO \\ INTERNACIONAL. \\ Disponível \\ em:}

[https://www.dgae.gov.pt/servicos/comercio-internacional-e-relacoes-

internacionais/multilaterais/organizacao-para-a-cooperacao-e-desenvolvimentoeconomico-ocde-.aspx]; acesso em 08 dez 2021.

PwC. Combate a fraudes e crimes econômicos: uma batalha sem fim Pesquisa Global sobre Fraudes e Crimes Econômicos 2020. Disponível em: [https://www.pwc.com.br/pt/publicacoes/servicos/assets/consultorianegocios/2020/pesquisa_sobre-fraudes-e-crimes-economicos-2020-pwc-brasil.pdf]; acesso em 20 out 2021.

SENNO, Elisângela Pereira; PRATES, Glaucia Aparecida; LUCENTE, Adriano dos Reis; GALLI, Lesley Carina do Lago Attadia; GALLI, Rafael Altafin. Benefícios da implantação do programa de Compliance em uma organização do setor de energia: um estudo de caso. II SITEFA - Simpósio de Tecnologia Fatec Sert5aozinho. Disponível em: [https://sitefa.fatecsertaozinho.edu.br/index.php/sitefa/article/view/90/51]; acesso em 08 dez 2021.

SIQUEIRA, Marina Paiva de. Cyber Risks: Fatores decisórios para a transferência mediante a contratação do seguro. Monografia [Bacharelado em Administração] apresentada ao Departamento de Administração da Universidade Federal Fluminense. Niterói, RJ. 2019.

RC: 103918

Disponibile in: https://www.nucleodoconhecimento.com.br/economiaaziendale/vantaggi-della-conformita 
SOBREIRA FILHO, Enoque Feitosa; LEITE, Flavia Piva Almeida; MARTINS, José Alberto Monteiro. Ética empresarial como base de sustentação do programa de Compliance: uma breve análise sobre a ética, a integridade $\mathrm{e} o$ Compliance. Revista Relações Internacionais no Mundo Atual. Vol. 2,n. 23, 2019. Disponível em: [http://revista.unicuritiba.edu.br/index.php/RIMA/article/view/3891/371373033]; acesso em 29 ago 2021.

SOUZA, Silvia Regina; MACIEL-LIMA, Sandra; LUPI, André Lipp Pinto Basto. Aplicabilidade do Compliance na Administração Pública em face ao momento político atual brasileiro. Percurso - Anais do I Congresso Ibro-Americano de Direito Empresarial e Cidadania (CONIBADEC), vol. 01, n. 24, Curitiba, p.1-22, 2018. Disponível em: [http://revista.unicuritiba.edu.br/index.php/percurso/article/view/3274/371371782]; acesso em 01 dez 2021.

3MIND JURÍDICO. Compliance: o que é e o benefício para empresas. Abril de 2021. Disponível em: [https://www.3mind.com.br/blog/compliance-empresas/]; acesso em 08 dez 2021.

VAZ, Tatiana. 5 empresas envolvidas em escândalos recentes. Artigo publicado em 30 abr 2012. Disponível em: [https://exame.com/negocios/5-empresas-envolvidasem-escandalos-recentes/]; acesso em 28 out 2021.

VEJA ON LINE. Funcionário de restaurante publica foto lambendo comida de clientes. Junho de 2013.2 Disponível em: [https://veja.abril.com.br/economia/funcionario-de-restaurante-publica-fotolambendo-comida-de-clientes/]; acesso em 14 out 2021.

Inviato: Novembre, 2021.

Approvato: Dicembre 2021.

RC: 103918

Disponibile in: https://www.nucleodoconhecimento.com.br/economiaaziendale/vantaggi-della-conformita 